Communications in Physics, Vol.20, No. 4 (2010), pp. 371-376

\title{
TRANSMITTANCE FUNCTION OF TWO-PORTS NONLINEAR FIBER MACH-ZEHNDER INTERFEROMETER
}

\author{
HO QUANG QUY \\ Academy of Military Science and Technology \\ NGUYEN THI THANH TAM \\ Vinh University, QuangNam University \\ NGUYEN VAN HOA \\ Hong Duc University
}

\begin{abstract}
In this article a two-ports nonlinear fiber Mach-Zehnder interferometer is proposed. The expression for output-input intensity relation and the transmittance function are derived based on principle operation of optical coupler. The expression of coupling coefficient of coupler is presented. The bell shape of the transmittance function is simulated. The results lead to investigate bistability of two-ports-nonlinear fiber Mach-Zehnder interferometer and to confirm bistable character of it in the future.
\end{abstract}

\section{INTRODUCTION}

Nowaday, the optical bistable device (OBD) is important in "photonics". They are used as an optical signal shaper, A/D converter, memory element, hard limiter, logic gate, etc $[1,2]$. Its pre-eminence quality belongs to bistability of output-input intensity relation. There are many kinds of OBD, whose operation is based on another principles, but most of them operate basing on principle of nonlinear interferometer $[2,6,7]$. The previous OBD are used active mediums as gas, liquid in cuvet, so their dimension is too big. With advantage in nano-semiconductor technology and optical fiber, the dimension of them is more and more reduced to nano-scale $[5,9,12]$. Up-to-date, the most useful OBD is nonlinear fiber Mach-Zehnder interferometer (NFM-ZI), which consist of a pair of fibers, one of them has nonlinear refrective index, the second has linear one. Two fibers couple one with other by two couplers (see Fig.1a). As shown in some works [3, 8], the transfer percentage of coupler depends on principle parameters as length of coupler, radius of fiber, and separate space between them. Moreover, the bistability of OBD depends also on the transfer percentage of coupler, input intensity, and the length of nonlinear fiber $[5,6,7,9]$. Thus, all above parameters influence on output intensity of OBD, i.e. on output-input intensity relation. This influence has still not been completely investigated. From Fig.1a, it is shown that the classical NFM-ZI has four ports: input, output, "feedback", and free. 


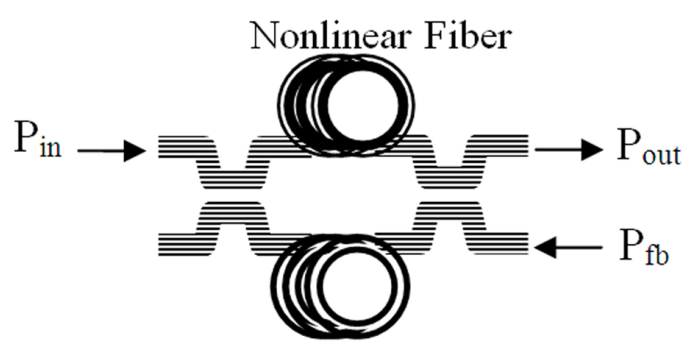

a

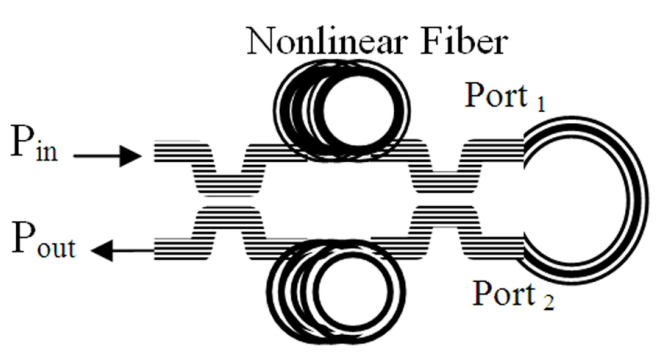

b

Fig. 1. (a) Four- ports NFM-ZI.

(b) two-ports NFM-ZI.

With this geometrical structure, it must be used two input light sources at the same time, strong one plays role of pump, and other weak plays role of "feedback" [1].

In this paper we propose a two-ports NFM-ZI (see Fig. 1b). In Sec. II, we derive equation discribing the output-input intensity relation. In Sec. III we present expression of transfer coefficient of coupler. In Sec. IV, we derive the transmittance function and simulate it.

\section{OUTPUT-INPUT INTENSITY RELATION}

Consider a two-ports NFM-ZI illustrated in Fig.1b. It consists of two couplers with transfer efficiencies, $\eta_{1}$ and $\eta_{2}[3,8]$, respectively, one nonabsorbing nonlinear fiber with nonlinear of refractive index coefficient, $\eta_{2}$ and length, $l_{n l}$, one nonabsorving linear fiber with length, $l_{l}$. Two ports of second coupler are connected by a linear fiber with length, $l_{\text {con }}$.

Denote $A_{\text {in }}$ be the amplitude of input light. The amplitude of light propagating through nonlinear fiber is given by [10]:

$$
A_{1}=\sqrt{\eta_{1}} A_{i n} e^{i \phi_{n l}}
$$

where $\phi_{n l}$ is the phase change after fiber distance of $l_{n l}$. The amplitude of light propagating through linear fiber is given by:

$$
A_{2}=\sqrt{1-\eta_{1}} A_{i n} e^{i \phi_{l}}
$$

where $\phi_{l}$ is the phase change after fiber distance of $l_{l}$. The amplitude of light propagating through second coupler at Port $_{1}$ is given by:

$$
A_{t o l, 1}=\sqrt{1-\eta_{1}} \sqrt{1-\eta_{2}} A_{i n} e^{i \phi_{l}}+\sqrt{\eta_{1}} \sqrt{\eta_{2}} A_{i n} e^{i \phi_{n l}},
$$

and at Port $_{2}$ is given by:

$$
A_{t o l, 2}=\sqrt{1-\eta_{1}} \sqrt{\eta_{2}} A_{i n} e^{i \phi_{l}}+\sqrt{\eta_{1}} \sqrt{1-\eta_{2}} A_{i n} e^{i \phi_{n l}} .
$$


From (3) and (4) the amplitude of feedback light from second coupler to nonlinear branch is:

$$
A_{f}=\left(\begin{array}{c}
\sqrt{\eta_{2}}\left[\sqrt{1-\eta_{1}} \sqrt{\eta_{2}} A_{i n} e^{i \phi_{l}}+\sqrt{\eta_{1}} \sqrt{1-\eta_{2}} A_{i n} e^{i \phi_{n l}}\right]+ \\
\sqrt{1-\eta_{2}}\left[\sqrt{1-\eta_{1}} \sqrt{1-\eta_{2}} A_{i n} e^{i \phi_{l}}+\sqrt{\eta_{1}} \sqrt{\eta_{2}} A_{i n} e^{i \phi_{n l}}\right]
\end{array}\right) e^{i \phi_{l c o n}},
$$

and the amplitude of light from second coupler to linear branch is:

$$
A_{l i n}=\left(\begin{array}{c}
\sqrt{1-\eta_{2}}\left[\sqrt{1-\eta_{1}} \sqrt{\eta_{2}} A_{i n} e^{i \phi_{l}}+\sqrt{\eta_{1}} \sqrt{1-\eta_{2}} A_{i n} e^{i \phi_{n l}}\right]+ \\
\sqrt{\eta_{2}}\left[\sqrt{1-\eta_{1}} \sqrt{1-\eta_{2}} A_{i n} e^{i \phi_{l}}+\sqrt{\eta_{1}} \sqrt{\eta_{2}} A_{i n} e^{i \phi_{n l}}\right]
\end{array}\right) e^{i \phi_{l c o n}} .
$$

From (5) and (6) the total amplitude of output light (goes outside from first coupler) is given by:

$$
\begin{aligned}
A_{\text {out }}= & \sqrt{1-\eta_{1}} A_{f}+\sqrt{\eta_{1}} A_{\text {lin }} \\
= & \sqrt{1-\eta_{1}}\left(\begin{array}{c}
\sqrt{\eta_{2}}\left[\sqrt{1-\eta_{1}} \sqrt{\eta_{2}} A_{i n} e^{i \phi_{l}}+\sqrt{\eta_{1}} \sqrt{1-\eta_{2}} A_{i n} e^{i \phi_{n l}}\right]+ \\
\sqrt{1-\eta_{2}}\left[\sqrt{1-\eta_{1}} \sqrt{1-\eta_{2}} A_{i n} e^{i \phi_{l}}+\sqrt{\eta_{1}} \sqrt{\eta_{2}} A_{i n} e^{i \phi_{n l}}\right]
\end{array}\right) e^{i \phi_{l c o n}}+ \\
& \sqrt{\eta_{1}}\left(\begin{array}{c}
\sqrt{1-\eta_{2}}\left[\sqrt{1-\eta_{1}} \sqrt{\eta_{2}} A_{i n} e^{i \phi_{l}}+\sqrt{\eta_{1}} \sqrt{1-\eta_{2}} A_{i n} e^{i \phi_{n l}}\right]+ \\
\sqrt{\eta_{2}}\left[\sqrt{1-\eta_{1}} \sqrt{1-\eta_{2}} A_{i n} e^{i \phi_{l}}+\sqrt{\eta_{1}} \sqrt{\eta_{2}} A_{i n} e^{i \phi_{n l}}\right]
\end{array}\right) e^{i \phi_{l c o n}},
\end{aligned}
$$

or

$$
A_{\text {out }}=A_{\text {lin }} e^{i \phi_{l c o n}} e^{i \phi_{l}}\left(\begin{array}{c}
\eta_{2}\left(1-\eta_{1}\right)+\left(1-\eta_{1}\right)\left(1-\eta_{2}\right)+2 \sqrt{\eta_{1} \eta_{2}\left(1-\eta_{1}\right)\left(1-\eta_{2}\right)}+ \\
{\left[\eta_{1}\left(1-\eta_{2}\right)+\eta_{1} \eta_{2}+2 \sqrt{\eta_{1} \eta_{2}\left(1-\eta_{1}\right)\left(1-\eta_{2}\right)}\right] e^{i\left(\phi_{n l}-\phi_{l}\right)}}
\end{array}\right) .
$$

From (8) the intensity of output light is given as follows:

$$
\begin{aligned}
I_{\text {out }} & =\frac{1}{2} \epsilon_{0} c\left|A_{\text {out }}\right|^{2} \\
& =I_{\text {in }}\left(\begin{array}{c}
{\left[\eta_{2}\left(1-\eta_{1}\right)+\left(1-\eta_{1}\right)\left(1-\eta_{2}\right)+2 \sqrt{\eta_{1} \eta_{2}\left(1-\eta_{1}\right)\left(1-\eta_{2}\right)}\right]^{2}+} \\
{\left[\eta_{1}\left(1-\eta_{2}\right)+\eta_{1} \eta_{2}+2 \sqrt{\eta_{1} \eta_{2}\left(1-\eta_{1}\right)\left(1-\eta_{2}\right)}\right]^{2}+} \\
2\left[\eta_{1}\left(1-\eta_{2}\right)+\eta_{1} \eta_{2}+2 \sqrt{\eta_{1} \eta_{2}\left(1-\eta_{1}\right)\left(1-\eta_{2}\right)}\right] \cos (\phi+\Delta \varphi)
\end{array}\right),
\end{aligned}
$$

where $\phi_{n l}-\phi_{l}=\phi+\Delta \phi, \phi$ is the phase shift of light in two branchs, $\Delta \varphi$ is initial phase shift of input light [11].

Now, we introduce the phase shift of two branchs. It is known that [4]

$$
\phi=\frac{2 \pi}{\lambda}\left(l_{n l}-l_{l}\right)
$$

where $l_{n l}, l_{l}$ are optical paths in nonlinear and linear branchs, respectively. Consider the physical length of fiber in two branchs are equal, $L$, so their optical paths are given by:

$$
l_{n l}=\left(n_{0}+n_{2} I_{c t r}\right) L, \quad l_{l}=n_{0} L .
$$

Substituting (11) into (10) we have:

$$
\phi=\frac{2 \pi}{\lambda} n_{2} I_{c t r} L
$$


where $I_{c t r}$ is the intensity of control light in nonlinear branch. As shown in work of Soljacic [12], the control intensity is a sum of average intensities of contributing lights, so from (1), (6) and (7) we have:

$$
\begin{aligned}
I_{c t r}= & \frac{I_{\text {out }}}{1-\eta_{1}}+I_{1}-\frac{\eta_{1} I_{\text {lin }}}{1-\eta_{1}} \\
= & \frac{I_{\text {out }}}{1-\eta_{1}}+I_{\text {in }} \eta_{1}+ \\
& I_{\text {in }}\left[\eta_{1}+4\left(1-\eta_{1}\right)\left(1-\eta_{2}\right) \eta_{2}+4 \sqrt{\left(1-\eta_{1}\right)\left(1-\eta_{2}\right) \eta_{1} \eta_{2} \cos (\phi+\Delta \varphi)}\right] \\
= & \frac{I_{\text {out }}}{1-\eta_{1}}+I_{\text {in }}\left[2 \eta_{1}+4\left(1-\eta_{1}\right)\left(1-\eta_{2}\right) \eta_{2}\right] .
\end{aligned}
$$
follows:

Substituting (13) and (12) into (9), the output-input intensity relation is given as

$$
I_{\text {out }}=I_{\text {in }}\left(\begin{array}{l}
{\left[\left(1-\eta_{1}\right)+2 \sqrt{\eta_{1} \eta_{2}\left(1-\eta_{1}\right)\left(1-\eta_{2}\right)}\right]^{2}+} \\
{\left[\eta_{1}+2 \sqrt{\eta_{1} \eta_{2}\left(1-\eta_{1}\right)\left(1-\eta_{2}\right)}\right]^{2}+2\left[\eta_{1}+2 \sqrt{\eta_{1} \eta_{2}\left(1-\eta_{1}\right)\left(1-\eta_{2}\right)}\right] \times} \\
\cos \left\{\frac{2 \pi n_{2} L}{\lambda\left(1-\eta_{1}\right)}\left[I_{\text {out }}+I_{\text {in }}\left(2\left(1-\eta_{1}\right) \eta_{1}+4\left(1-\eta_{1}\right)^{2}\left(1-\eta_{2}\right) \eta_{2}\right)\right]+\Delta \varphi\right.
\end{array}\right) .
$$

\section{TRANSFER COEFFICIENT OF FIBER COUPLER}

Consider a fiber coupler with principle parameters as illustrated in Fig.2. Its transfer coefficient is given by:

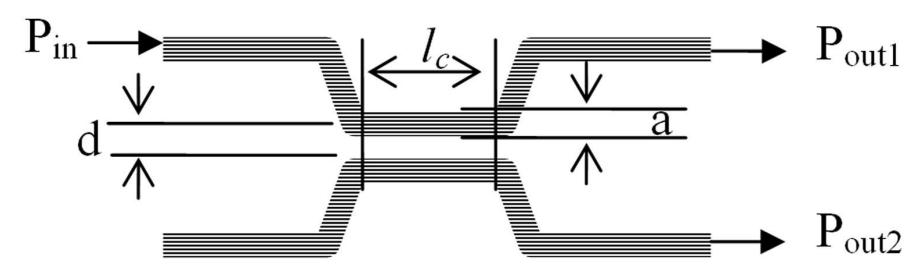

\section{Linear Fiber Coupler}

Fig. 2. Schematic of fiber coupler.

$$
\eta=\frac{P_{\text {out } 1}\left(l_{c}\right)}{P_{\text {in }}(0)}=\cos ^{2}\left(C l_{c}\right), \quad 1-\eta=\frac{P_{\text {out } 2}\left(l_{c}\right)}{P_{\text {in }}(0)}=\sin ^{2}\left(C l_{c}\right),
$$

where $C$ is the coupling parameter for two fibers with radius $a$, and separate space $d$, refractive indexes $n_{1}$ and $n_{2}$, which can be approximatively given by [8]:

$$
C=\frac{\pi}{2} \frac{\sqrt{\delta}}{a} \exp \left\{-\left(A+B \frac{d}{a}+D \frac{d^{2}}{a^{2}}\right)\right\}
$$




$$
\begin{aligned}
& \text { Where } \\
& \qquad \begin{array}{l}
\delta=\frac{n_{1}^{2}-n_{2}^{2}}{n_{1}^{2}} ; A=5.2789-3.6631 V+0.3841 V^{2} ; B=0.7769-1.2252 V+0.3841 V^{2} ; \\
D=-0.0175-0.0064 V-0.0009 V^{2} ; \quad V=\frac{2 \pi a \sqrt{n_{1}^{2}-n_{2}^{2}}}{\lambda_{0}} .
\end{array}
\end{aligned}
$$

From (14) to (17) we can see that the output intensity of two-ports NFI depends not only on typical parameters as input intensity, length of nonlinear fiber, nonlinear refractive index coefficient, initial phase shift, but also on principle parameters of coupler as radius, separate space, refractive index, and length.

\section{TRANSMITTANCE FUNCTION}

Puting a normalized intensity, $X=\frac{n_{2} L I}{\lambda}$, the Eq.(14) is rewritten as follows:

$$
X_{\text {out }}=X_{\text {in }}\left(\begin{array}{l}
{\left[\left(1-\eta_{1}\right)+2 \sqrt{\eta_{1} \eta_{2}\left(1-\eta_{1}\right)\left(1-\eta_{2}\right)}\right]^{2}+} \\
{\left[\eta_{1}+2 \sqrt{\eta_{1} \eta_{2}\left(1-\eta_{1}\right)\left(1-\eta_{2}\right)}\right]^{2}+2\left[\eta_{1}+2 \sqrt{\eta_{1} \eta_{2}\left(1-\eta_{1}\right)\left(1-\eta_{2}\right)}\right] \times} \\
\cos \left\{\frac{2 \pi}{1-\eta_{1}}\left[X_{\text {out }}+X_{\text {in }}\left(2\left(1-\eta_{1}\right) \eta_{1}+4\left(1-\eta_{1}\right)^{2}\left(1-\eta_{2}\right) \eta_{2}\right)\right]+\Delta \varphi\right\}
\end{array}\right) .
$$

From this equation, the basic equation for bistable device $X_{\text {in }}=\frac{X_{\text {out }}}{\Im\left(X_{\text {out }}\right)}$ can be determined with the transmittance function is given by:

$$
\Im\left(X_{\text {out }}\right)=\left(\begin{array}{l}
{\left[\left(1-\eta_{1}\right)+2 \sqrt{\eta_{1} \eta_{2}\left(1-\eta_{1}\right)\left(1-\eta_{2}\right)}\right]^{2}+} \\
{\left[\eta_{1}+2 \sqrt{\eta_{1} \eta_{2}\left(1-\eta_{1}\right)\left(1-\eta_{2}\right)}\right]^{2}+2\left[\eta_{1}+2 \sqrt{\eta_{1} \eta_{2}\left(1-\eta_{1}\right)\left(1-\eta_{2}\right)}\right] \times} \\
\cos \left\{\frac{2 \pi}{1-\eta_{1}}\left[X_{\text {out }}+X_{\text {in }}\left(2\left(1-\eta_{1}\right) \eta_{1}+4\left(1-\eta_{1}\right)^{2}\left(1-\eta_{2}\right) \eta_{2}\right)\right]+\Delta \varphi\right\}
\end{array}\right) .
$$

Figure 3 presents an example simulated to show transmittance function. The bell shape, i.e. the principle shape of transmittance function gives to arise bistability of twoports NFM-ZI, appears in suitable range of output intensity. In the range of $I_{\text {out }}$ from 0 to $8.10^{8} \mathrm{~W} / \mathrm{cm}^{2}$ (Fig.3a) there are three critical points (marked by cycles), every of them is a state-jump point. In the range of $I_{\text {out }}$ from 7.5 to $15.10^{8} \mathrm{~W} / \mathrm{cm}^{2}$ (Fig. $3 \mathrm{~b}$ ), there are other three state-jump points.

Such, from (14), (16), (19), and Fig. 3, we can see that two-ports NFM-ZI has bistable character and will operate as a bistable device with suitable input intensity.

\section{CONCLUSION}

The expressions of the output-input intensity relation and the transmittance function for two-ports NFM-ZI are derived. The simulated bell shape of the transmittance function result in that the proposed two-ports NFM-ZI has bistable character and will operate as a bistable device. The bistability of it will be investigated in the next work to 

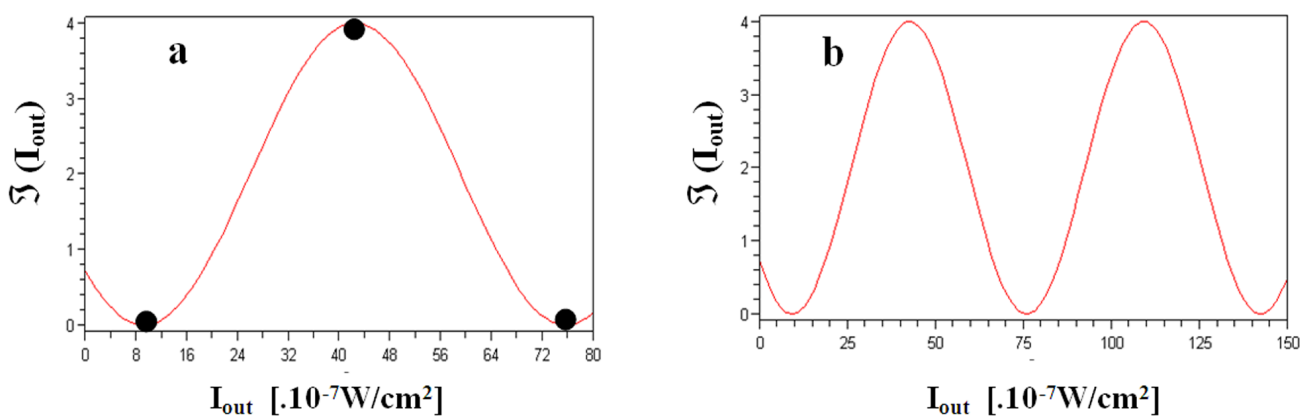

Fig. 3. Transimittance function $\Im\left(I_{\text {out }}\right)$.
a: $I_{\text {out }}=\left(0 \div 8.10^{8}\right) \mathrm{W} / \mathrm{cm}^{2}$.
b: $I_{\text {out }}=\left(0 \div 15.10^{8}\right) \mathrm{W} / \mathrm{cm}^{2}$

with $\mathrm{L}=100 \mathrm{~mm}, n_{n l}=10^{-14} \mathrm{~cm}^{2} / W, I_{i n}=10^{10} \mathrm{~W} / \mathrm{cm}^{2}, \eta_{1}=\eta_{2}=0.5, \Delta \varphi=-0.06 \pi$.

confirm the above conclusion. Moreover, the bistability of two-ports NFM-ZI depends not only on input intensity, but on coupler's transfer coefficient, which depends on principle parameters of coupler. It means that it needs to investigate in detail the influence of all principle parameters on bistability of two-ports NFM-ZI, before designing it.

\section{REFERENCES}

[1] M. C. D. Andrew, Opt. Switching, OPN (2005) 34.

[2] L. Brzozowski et al., J. of Lightwave Technology, 19 (2001) 114-119.

[3] M. Cai et al., Opt. Letter, 25, (2000) 1430.

[4] W. Demtroder, Laser spectroscopy, (1982).

[5] A. Erlacher et al., J. of Appl.Phys, 95, (2004) 2927-2929.

[6] N. V. Hoa, H. Q. Quy, and V. N. Sau, Commun. in Phys, 15, (2005) 223-228.

[7] N. V. Hoa, H. Q. Quy, and D. X. Khoa, Proc. of GVS6, Chemnitz, (2003) 144-147.

[8] J. M. Jonathan, Summer School, Doson, (2004) 245.

[9] H. T. Martin et al., Otp. Tech. Letter 31 (2001) 411-415.

[10] H. Q. Quy, V. N. Sau, and N. V. Hoa, Commun. in Phys. 13 (2003) 157-164.

[11] H. Sakata, Appl. Phys, 40, (2001) 240-248.

[12] M. Soljacic et al., Phys. Rev. E66 (2002) 05560-1-05560-4(R).

Received 12 September 2009. 